\title{
THE OPTIMAL CONTROL LANDSCAPE FOR THE GENERATION OF UNITARY TRANSFORMATIONS WITH CONSTRAINED DYNAMICS
}

\author{
MICHAEL HSIEH ${ }^{1,2}$, REBING WU $^{5}$, HERSCHEL RABITZ ${ }^{6}$, DANIEL LIDAR $^{1,2,3,4}$ \\ ${ }^{1}$ CENTER FOR QUANTUM INFORMATION SCIENCE AND TECHNOLOGY, ${ }^{2}$ DEPARTMENT OF CHEMISTRY, \\ ${ }^{3}$ DEPARTMENT OF ELECTRICAL ENGINEERING, ${ }^{4}$ DEPARTMENT OF PHYSICS, UNIVERSITY OF \\ SOUTHERN CALIFORNIA, LOS ANGELES, CA 90089, ${ }^{5}$ DEPARTMENT OF AUTOMATION, TSINGHUA \\ UNIVERSITY, BEIJING, 100084, P.R. CHINA, ${ }^{6}$ DEPARTMENT OF CHEMISTRY, PRINCETON UNIVERSITY, \\ PRINCETON, NJ 08544
}

\begin{abstract}
The reliable and precise generation of quantum unitary transformations is essential to the realization of a number of fundamental objectives, such as quantum control and quantum information processing. Prior work has explored the optimal control problem of generating such unitary transformations as a surface optimization problem over the quantum control landscape, defined as a metric for realizing a desired unitary transformation as a function of the control variables. It was found that under the assumption of non-dissipative and controllable dynamics, the landscape topology is trap-free, implying that any reasonable optimization heuristic should be able to identify globally optimal solutions. The present work is a control landscape analysis incorporating specific constraints in the Hamiltonian corresponding to certain dynamical symmetries in the underlying physical system. It is found that the presence of such symmetries does not destroy the trap-free topology. These findings expand the class of quantum dynamical systems on which control problems are intrinsically amenable to solution by optimal control.
\end{abstract}

\section{InTRODUCTION}

Central to many problems in quantum control [1] and quantum information processing [2] is the stable and precise generation of specific unitary transformations. This task may be viewed as an inverse problem where given a desired unitary transformation, one must obtain the values of the control variables of the system Hamiltonian whose dynamics generate it.

Date: October 30, 2018. 
Generally, the connection between the control variables and the unitary evolution operator is sufficiently complex such that the exact control solution cannot be deduced from first principles. In such cases adaptive optimization techniques are commonly applied, such as optimal control theory (OCT) methods based on classical variational optimization [3] for computer simulations [4] and optimal control experiment (OCE) methods based on evolutionary adaptation [5] for practical laboratory studies [6]. A method for the optimal generation of unitary transformations based on OCT methods has been recently introduced [7, with some promising successful applications in simulation studies [8].

A basic question is why such methods have had such a surprising degree of success in such complex, high-dimensional problems. A step toward the resolution of this question has been found in recent theoretical studies of the quantum control landscape [9, 10, defined generally as the metric of attainment of some optimization objective as a function of the control variables. In the present case, the optimization objective is the generation of a specific unitary transformation of the quantum system.

Consider a controllable quantum system defined on an $N$-level Hilbert space whose dynamics are given by the time-dependent Hamiltonian $H(t)$. The unitary evolution operator for the system is

$$
S\left(t_{i}, t_{f}\right)=\mathbf{T}_{+} \exp \left[-\frac{i}{\hbar} \int_{t_{i}}^{t_{f}} d t H(t)\right]
$$

where $\mathbf{T}_{+}$denotes the time-ordering operation over some time interval $\left[t_{i}, t_{f}\right]$. The optimization problem entails choosing the controls within $H(t)$ that steer $S\left(t_{i}, t_{f}\right)$ to some desired target transformation $W\left(t_{i}, t_{f}\right) \equiv W$. We assume a degree of system controllability such that for any $S\left(t_{i}, t_{f}\right) \equiv S$ selected from the unitary Lie group $U(N)$, there exists some choice of controls within $H(t)$ which generates $S$ via Eq.(11). This full access to $U(N)$ makes it possible to define the control variables of the problem to be some parametrization of $S$ itself, in lieu of the controls within $H(t)$. This kinematic representation of the control variables is particularly attractive in the sense that the analysis now depends no longer on the specific structure of the Hamiltonian, but only on the geometry of $U(N)$. Furthermore, the results are generalizable to all finite-dimensional, controllable quantum systems.

We employ as the distance measure between unitary transformations the squared Hilbert-Schmidt metric $\|S-W\|^{2}=2 N-2 \operatorname{Re} \operatorname{Tr}\left(W^{\dagger} S\right)$, whose nonconstant part we define to be the landscape metric 
function

$$
J[S]=\operatorname{Re} \operatorname{Tr}\left(W^{\dagger} S\right)
$$

Trace functionals of this type have been studied in a general context [11, 12, as well as with specific reference to physical [13] and control theoretic applications [14, 15. We define the control landscape to be the image of the metric function $J: U(N) \rightarrow \mathbb{R}$, where each point in $U(N)$ represents some choice of controls. We will refer to the space over which the landscape is defined as the landscape domain, which in this instance is $U(N)$.

The optimal control problem of generating unitary transformations of a desired form is essentially a search over the control landscape for critical regions at which the first-order variation of the landscape function $J$ vanishes. In this sense, the difficulty of the problem is largely determined by the topology of the critical points. In a prior analysis of the problem of optimally generating unitary transformations over $U(N)$ [10, it was found that the number of disconnected critical regions of the landscape corresponding to distinct values of $J$ scaled as $N+1$, of which $N-1$ regions corresponded to local critical points with saddlepoint topology, and the remaining to global extrema. Furthermore, it was determined that the global extrema solutions comprised zero-dimensional subspaces of $U(N)$, where the local critical regions had the structure of complex Grassmannian submanifolds embedded in $U(N)$ [10].

Although the existence of such local (false) critical points may still act as deleterious attractors for optimal searches, the number of such regions grow only linearly with the Hilbert space dimension of the underlying physical system. These appear to be competing arguments in the assessment of whether this landscape is amenable to adaptive searching methods. In recent numerical studies where genetic algorithms, gradient following, and simplex methods have been used, the observed exponential scaling of search convergence time suggests that the deleterious influences may dominate [16]. Nevertheless, the desirable absence of any local extremum traps over the entire class of such surfaces assures that any optimization will eventually succeed in locating a global solution.

A key open question is whether this desirable trap-free landscape topology is preserved when the underlying dynamics of the physical system are restricted or constrained in some manner. In the present 
analysis, we consider various cases in which specific dynamical symmetries are imposed on the system Hamiltonian, specifically relating to total spin, space rotation invariance, and time-reversal invariance. In prior analyses [9, 10, no structure of the Hamiltonian was assumed other than its complex-valued Hermiticity (i.e., the imaginary part of the Hamiltonian is nonzero), which corresponds to the symmetry class of systems without time-reversal invariance [17. We presently consider the topology of the control landscape defined over physical systems with Hamiltonians from some alternate symmetry classes:

(1) Systems with time-reversal invariance and integral total spin.

(2) Systems with time-reversal invariance and space rotation symmetry.

(3) Systems with with time-reversal invariance, half-integer total spin, without space rotation symmetry.

The first two classes are described by real symmetric Hamiltonians $H=H^{T}$. Such Hamiltonians arise in time-symmetric pulsing strategies in spin control problems [18, 19, 20, and continuous quantum random walks [21, 22]. The third class is described by symplectic Hamiltonians $H=H^{R}$, where the $2 N \times 2 N$ matrix $H^{R}$ is the symplectic dual

$$
H^{R} \equiv-\left(\begin{array}{rr} 
& \mathbb{I}_{N} \\
-\mathbb{I}_{N} &
\end{array}\right) H^{T}\left(\begin{array}{rr} 
& \mathbb{I}_{N} \\
-\mathbb{I}_{N} &
\end{array}\right)
$$

Such Hamiltonians arise in the dynamics of Gaussian pure states [23], which have notable applications in quantum optics [24]. A more extensive discussion connecting symmetry classes of Hamiltonians with their corresponding physical systems can be found in [25, 26]

Section 2 presents formal definitions of the landscape function and domain for the case of real symmetric Hamiltonians. From these definitions, the remarkable property of the invariance of the landscape topology with respect to the choice of target transformation follows simply. The identification of the critical landscape regions as a union of real Grassmannian submanifolds is given in Section 3. The method for computing the signature of the critical submanifolds is derived in Section 4. Section 5 recapitulates the analysis for the case of symplectic Hamiltonians. Section 6 concludes. 


\section{Landscape Function and Domain}

Consider the class of physical systems whose dynamics are described by real symmetric Hamiltonians. Through Eq.(11), the dynamical propagators generated by such Hamiltonians are symmetric unitary transformations. Let $S$ be a programmable $N \times N$ symmetric unitary transformation satisfying (i) $S S^{\dagger}=S^{\dagger} S=I_{N}$, where $I_{N}$ is the $N \times N$ identity, and (ii) $S=S^{T}$. These constraints leave $\frac{N^{2}+N}{2}$ real degrees of freedom in $S[26$. For some desired symmetric unitary transformation $W$, the optimization objective is to minimize the landscape metric distance between $W$ and $S$ as defined in Eq.(2). In general, optimization heuristics defined over the landscape will seek the critical points of the landscape where the first-order variation of the landscape metric function $J$ vanishes. A remarkable quality of this optimization problem is that the landscape topology is invariant to the choice of the target transformation $W$. In this sense, the optimization problems for all unitary transformations can be expected to be of equivalent difficulty. We presently prove this claim.

We assume that the range of controls is restricted such that the Hamiltonian generating $S$ is always real symmetric. Any symmetric unitary transformation $S$ has the canonical representation $S=U^{T} U$ where $U \in U(N)\left[26\right.$. Let $U_{1}, U_{2} \in U(N)$. The necessary and sufficient condition for $U_{1}^{T} U_{1}=U_{2}^{T} U_{2}$ is that $U_{1} U_{2}^{-1} \in O(N)$ where $O(N)$ denotes the real orthogonal Lie group. Therefore, the image of the canonical representation mapping $U \rightarrow U^{T} U$ is homeomorphic to the homogeneous space $U(N) / O(N)$ [27. Henceforth, we take the space $U(N) / O(N)$ of symmetric unitary transformations to be the landscape domain.

Since $U(N) / O(N)$ is not closed under multiplication, we must rearrange the argument of the trace function $J[S]=\operatorname{Re} \operatorname{Tr}\left(\sqrt{W^{\dagger}} S \sqrt{W^{\dagger}}\right)$, where $S$ and $W$ are symmetric unitary, to ensure that the landscape metric is defined strictly over the landscape domain. Noting that $\sqrt{W^{\dagger}} S \sqrt{W^{\dagger}} \rightarrow S$ is a homeomorphism on $U(N) / O(N)$, the image of $J[\cdot]=\operatorname{Re} \operatorname{Tr}(\cdot)$ with the choice of $S$ as the argument is equivalent to that with the choice of $\sqrt{W^{\dagger}} S \sqrt{W^{\dagger}}$. Adopting the simpler choice of $S$ as the argument, we obtain the topologically equivalent landscape metric function

$$
\mathcal{J}[S]=\operatorname{Re}_{5} \operatorname{Tr}(S)
$$


which has no dependence on the target transformation $W$. This target-invariant landscape function $\mathcal{J}$ also has the advantage of analytical simplicity, which we adopt for the remainder of the analysis.

\section{Critical Submanifolds}

We presently determine the enumeration and topology of the landscape regions on which $\mathcal{J}$ is critical. We will demonstrate that the number of such regions scales linearly with $N$ and has the structure of real Grassmannians embedded in $U(N) / O(N)$.

Any symmetric unitary transformation can be diagonalized as

$$
S=X^{T} \Omega X
$$

where $X$ is an element of the real special orthogonal Lie group $S O(N)$ and $\Omega$ is a diagonal operator

$$
\Omega=\left(\begin{array}{ccc}
e^{i \varphi_{1}} & & \\
& \ddots & \\
& & e^{i \varphi_{N}}
\end{array}\right)
$$

of the unimodular eigenvalues $\left\{e^{i \varphi_{1}}, \ldots, e^{i \varphi_{N}}\right\}$ of $S[28]$. Using the cyclic property of the trace, the target-invariant landscape metric function simplifies to

$$
\begin{aligned}
\mathcal{J}[S] & =\operatorname{Re} \operatorname{Tr}(\Omega) \\
& =\sum_{j=1}^{N} \cos \varphi_{j} .
\end{aligned}
$$

From Eq.(8), we see that the first-order variation $\delta \mathcal{J}=-\sum_{j=1}^{N} \sin \varphi_{j} d \varphi_{j}$ vanishes when $\varphi_{j}=\ell_{j} \pi$, for any integers $\ell_{j}$. There are $N+1$ critical values $-N,-N+2, \ldots, N-2, N$ for $\mathcal{J}$, determined by the parities of $\ell_{j}, j=1, \ldots, N$. The corresponding critical points $\tilde{S}=X^{T} \tilde{\Omega}^{(n)} X$ comprise equivalence classes of transformations orthogonally similar to canonical elements $\tilde{\Omega}^{(n)}$ where $n$ denotes the number of even integers in the set $\left\{\ell_{1}, \ldots, \ell_{N}\right\}$ :

$$
\tilde{\Omega}^{(n)}=\left(\begin{array}{cc}
\mathbb{I}_{n} & \\
& -\mathbb{I}_{N-n}
\end{array}\right) .
$$


We seek to determine the topology of the $N+1$ equivalence classes of transformations corresponding to each distinct critical value. Such equivalence classes are the critical submanifolds of the landscape, composed of points at which the first-order optimization condition $\nabla \mathcal{J}=0$ is satisfied.

Consider the conjugation of $\tilde{\Omega}^{(n)} \in U(N) / O(N)$ by some $\Xi \in S O(N)$ as a group action $\mathcal{G}: S O(N) \times$ $U(N) / O(N) \rightarrow U(N) / O(N)$

$$
\mathcal{G} \cdot \tilde{\Omega}^{(n)}=\Xi^{T} \tilde{\Omega}^{(n)} \Xi
$$

for which $S O(N)$ is the acting group and $U(N) / O(N)$ is the $\mathcal{G}$-space. The stabilizer (isotropy) subgroup $\operatorname{STAB}\left(\tilde{\Omega}^{(n)}\right)$ of any $\tilde{\Omega}^{(n)}$, defined as the subgroup of the acting group $S O(N)$ whose elements map $\tilde{\Omega}^{(n)}$ back to itself via the $\mathcal{G}$-action, is composed exclusively of elements of the form

$$
\tilde{\Xi}=\left(\begin{array}{cc}
\Xi_{n} & \\
& \Xi_{N-n}
\end{array}\right)
$$

where $\Xi_{n} \in S O(n)$ and $\Xi_{N-n} \in S O(N-n)$. Therefore, the stabilizer is a product subgroup of $S O(N)$ :

$$
\operatorname{STAB}\left(\tilde{\Omega}^{(n)}\right)=S O(n) \times S O(N-n)
$$

Define a mapping which associates a fixed $\mathcal{G}$-space element $\Omega$ with some element of the acting group:

$$
\mathcal{G}_{\Omega}: S O(N) \rightarrow U(N) / O(N), \quad \Omega \rightarrow \mathcal{G} \cdot \Omega
$$

When the domain of $\mathcal{G}_{\Omega}$ is taken to be all of $S O(N)$, the image is simply the orbit of $\Omega$ :

$$
\mathcal{O}(\Omega) \equiv\left\{\Xi^{T} \Omega \Xi: \Xi \in S O(N)\right\}
$$

These orbits, for $\Omega=\tilde{\Omega}^{(n)}$, where $n=0, \ldots, N$, comprise precisely the $N+1$ sets of critical points of the landscape.

To obtain their topological structure, we recall that by the orbit-stabilizer theorem, $\mathcal{G}_{\Omega}$ induces a bijection $S O(N) / \operatorname{STAB}\left(\tilde{\Omega}^{(n)}\right) \rightarrow \mathcal{O}\left(\tilde{\Omega}^{(n)}\right)$, which we may sharpen further to be a diffeomorphism because $S O(N)$ is a compact Lie group [29. Therefore, the critical set is composed of the union

$$
\bigcup_{n=0}^{N} G_{\text {real }}(n, N)
$$


where

$$
G_{\text {real }}(n, N)=S O(N) / S O(n) \times S O(N-n)
$$

embedded in $U(N) / O(N)$. The dimensionality of the Grassmannian is well known to be

$$
\begin{aligned}
\operatorname{dim} G_{\text {real }}(n, N) & =\frac{N^{2}-N}{2}-\left[\frac{n^{2}-n}{2}+\frac{(N-n)^{2}-N+n}{2}\right] \\
& =n(N-n) .
\end{aligned}
$$

Since the objective of any optimization is to attain the $\mathcal{J}=N$ or $-N$ values, corresponding to a perfect generation of the desired transformation, an immediate consequence of the foregoing dimensionality equation is that the critical submanifolds corresponding to non-global critical have nonzero dimension in appropriate subspaces of $U(N) / O(N)$ whereas the critical submanifolds corresponding to global critical points, with $n=0$ or $n=N$, strictly have dimension zero. It is therefore of practical importance to determine whether the critical submanifolds corresponding to non-global critical points have the topology of local maxima or minima, which may act as traps, or of non-trapping saddlepoints.

\section{Hessian Analysis of Critical Points}

A critical point of $\mathcal{J}$ can be identified as an extremum or a saddlepoint by computing its signature $\left(D_{+}, D_{-}, D_{0}\right)$, an ordered triple of integers denoting the number of upward, downward and flat landscape directions at that point. $D_{+}$and $D_{-}$are commonly referred to as the indices of positive and negative inertia, and $D_{0}$ as the kernel dimension. The Hessian operator of $\mathcal{J}$ evaluated at $\tilde{S}$ is defined

$$
\mathcal{H}_{i^{\prime} i}=\frac{d^{2} \mathcal{J}[\tilde{\mathcal{S}}]}{d x_{i^{\prime}} d x_{i}}
$$

where $\left\{x_{i}\right\}_{i=1, \ldots, \frac{N^{2}+N}{2}}$ is some set of local coordinates around a point $S \in U(N) / O(N)$. Since the Hessian is symmetric, there exists some coordinate transformations $x_{i} \rightarrow \hat{x}_{i}$ which rotates $\mathcal{H}$ into diagonal form, where the enumeration of its positive, negative and zero-valued eigenvalues corresponds exactly to $D_{+}, D_{-}$and $D_{0}$. By Sylvester's Law of Inertia, $D_{+}, D_{-}$and $D_{0}$ are invariant to changes in the coordinate system $[30$. 
As a symmetric matrix, $\mathcal{H}$ is representable as a quadratic form $\langle\Gamma|Q(\mathcal{H})| \Gamma\rangle=\sum_{i, j} Q_{i, j} \Gamma_{i} \Gamma_{j}$, with real coefficients $\left\{Q_{i, j}\right\}$ and $\left\{\Gamma_{i}, \Gamma_{j}\right\}$ denoting components of a vector $|\Gamma\rangle$ of real indeterminates. A corollary of Sylvester's Law assures that the coordinate rotation $x_{i} \rightarrow \hat{x}_{i}$ induces a transformation of the quadratic form into a canonical form of strictly second degree monomials

$$
\langle\Gamma|\hat{Q}(\mathcal{H})| \Gamma\rangle=\sum_{i} \hat{Q}_{i} \hat{\Gamma}_{i}^{2}
$$

where the enumeration of the positive, negative and zero-valued real coefficients $\hat{Q}_{j}$ corresponds to $D_{+}, D_{-}$and $D_{0}[30$.

To explicitly connect the Hessian matrix with its quadratic form, let us consider $\mathcal{J}$ as a mapping $(\mathcal{J} \circ \gamma)(t)$ over a parameterized $\operatorname{arc} \gamma(t) \in U(N) / O(N)$ satisfying $\gamma(0)=\tilde{S}$. Taking the second derivative of the arc-parameterized $\mathcal{J}$ at $t=0$, we have 31.

$$
\begin{aligned}
(\mathcal{J} \circ \gamma)^{\prime \prime}(0) & =\left(\sum_{i} \frac{\partial \mathcal{J}[\gamma(0)]}{\partial x_{i}} \dot{\gamma}_{i}(0)\right)^{\prime} \\
& =\sum_{i} \frac{\partial \mathcal{J}[\gamma(0)]}{\partial x_{i}} \ddot{\gamma}_{i}(0)+\sum_{i, j} \frac{\partial^{2} \mathcal{J}[\gamma(0)]}{\partial x_{i} \partial x_{j}} \dot{\gamma}_{i}(0) \dot{\gamma}_{j}(0)
\end{aligned}
$$

Noting that $\frac{\partial \mathcal{J}[\gamma(0)]}{\partial x_{i}}$ vanishes since $\tilde{S}$ is critical, we identify the remaining term $\sum_{i, j} \frac{\partial^{2} \mathcal{J}[\gamma(0)]}{\partial x_{i} \partial x_{j}} \dot{\gamma}_{i}(0) \dot{\gamma}_{j}(0)$ as a quadratic form mapping tangent space vectors $\dot{\gamma}(0) \in T_{\tilde{S}} U(N) / O(N)$ to the reals [31]. Transforming into the diagonal coordinate system $\left\{\hat{x}_{i}\right\}$, we have

$$
(\mathcal{J} \circ \gamma)^{\prime \prime}(t)=\sum_{i} \frac{\partial^{2} \mathcal{J}[\gamma(t)]}{\partial \hat{x}_{i}^{2}} \dot{\gamma}_{i}^{2}(0)
$$

Associating $\frac{\partial^{2} \mathcal{J}[\gamma(t)]}{\partial \hat{x}_{i}^{2}} \leftrightarrow \hat{Q}_{i}$ and $\gamma_{i}^{2}(0) \leftrightarrow \hat{\Gamma}_{i}^{2}$, we obtain a direct identification of the Hessian matrix with its quadratic form.

We now compute the Hessian quadratic form (HQF) explicitly. Let us evaluate $\mathcal{J}$ at some critical point $\tilde{S}$ in the previously defined representation $\tilde{S}=X^{T} \tilde{\Omega} X$. Since $\tilde{S}$ is a critical point of $\mathcal{J}, \tilde{\Omega}$ takes the form

$$
\tilde{\Omega}=\left(\begin{array}{ccc}
\tilde{\omega}_{1} & & \\
& \ddots & \\
& & \tilde{\omega}_{N}
\end{array}\right) \text {. }
$$


where $\tilde{\omega}_{1}= \pm 1, \ldots, \tilde{\omega}_{N}= \pm 1$. If $\tilde{\omega}_{j}=+1(-1)$ for all $j$, we are at a global maximum (minimum). Otherwise, we are at a local critical point. We presently seek to establish that these critical points are not local extremum traps.

To obtain the HQF, we twice differentiate the landscape function argument $S$ along the parameterized curve $S=\sqrt{\tilde{S}} e^{i A t} \sqrt{\tilde{S}}$ in $U(N) / O(N)$ defined by some real matrix of indeterminates $A=A^{T}$ :

$$
S \rightarrow S^{\prime}=\left.\frac{d}{d t}\right|_{t=0} \sqrt{\tilde{S}} e^{i A t} \sqrt{\tilde{S}}=\sqrt{\tilde{S}} i A \sqrt{\tilde{S}} \rightarrow S^{\prime \prime}=-\sqrt{\tilde{S}} A^{2} \sqrt{\tilde{S}}
$$

and evaluate $\mathcal{J}$ explicitly in terms of the matrix elements of $A$.

$$
\begin{aligned}
\mathcal{H}_{\mathcal{J}}(S) & \equiv-\operatorname{Re} \operatorname{Tr}\left(A^{2} S\right) \\
& =-\operatorname{Re} \operatorname{Tr}\left(X A^{2} X^{T} \tilde{\Omega}\right) \\
& =-\operatorname{Re} \operatorname{Tr}\left(\tilde{A}^{2} \tilde{\Omega}\right) \\
& =-\sum_{i \neq j} \tilde{\omega}_{i} \tilde{A}_{i j}^{2}-\sum_{i} \tilde{\omega}_{i} \tilde{A}_{i i}^{2} \\
& =-\sum_{i<j}\left(\tilde{\omega}_{i}+\tilde{\omega}_{j}\right) \tilde{A}_{i j}^{2}-\sum_{i} \tilde{\omega}_{i} \tilde{A}_{i i}^{2},
\end{aligned}
$$

where $\tilde{A} \equiv X A X^{T}$ and multiplicative constants have been normalized to unity.

To determine the values of $D_{+}, D_{-}, D_{0}$, we note that for a critical point $\tilde{S}$ with eigenvalues +1 and -1 with multiplicities $n$ and $N-n$, the only nonzero coefficients in the first summation $-\sum_{i<j}\left(\tilde{\omega}_{i}+\tilde{\omega}_{j}\right) \tilde{A}_{i j}^{2}$ of Eq.(30) will be generated by $\tilde{\omega}_{i}=\tilde{\omega}_{j}=+1$ or $\tilde{\omega}_{i}=\tilde{\omega}_{j}=-1$. There are $\frac{n(n-1)}{2}$ ways of selecting indices $i$ and $j$ satisfying $i<j$ from the $n$-fold set of indices corresponding to positive values $\left\{k: \tilde{\omega}_{k}=+1\right\}$, and thus $\frac{n(n-1)}{2}$ negative-valued monomials $\tilde{A}_{i j}^{2}$ in this summation. We add this to the $n$ additional negative monomials from the second summation $-\sum_{i} \tilde{\omega}_{i} \tilde{A}_{i i}^{2}$ to obtain $D_{-}=\frac{n^{2}+n}{2}$. By similar counting, it can be seen that there are $D_{+}=\frac{(N-n)^{2}+N-n}{2}$ positive monomials. Noting that the sum of the indices of inertia and the kernel dimension must equal the dimensionality of the domain of the function whose 
Hessian is being computed

$$
\begin{aligned}
D_{+}+D_{-}+D_{0} & =\operatorname{dim} U(N) / O(N) \\
& =\frac{N^{2}+N}{2},
\end{aligned}
$$

it is evident that

$$
\begin{aligned}
& D_{+}=\frac{(N-n)^{2}+N-n}{2} \\
& D_{-}=\frac{n^{2}+n}{2} \\
& D_{0}=n(N-n) .
\end{aligned}
$$

Finally, it is evident that for any local critical point for which both $N, N-n>0$, both $D_{+}$and $D_{-}$must be nonzero. Therefore, all local critical points are assured to possess saddlepoint topology, and cannot act as local extremum traps.

\section{Control Landscape Topology for Symplectic Hamiltonians}

We consider the class of physical systems whose dynamics are described by symplectic Hamiltonians. Since the analysis is almost identical to the real symmetric Hamiltonian case, the discussion will be limited to statements of the key assumptions and conclusions. Let $S$ denote programmable symplectic unitary dynamical operator generated by such a Hamiltonian via Eq.(1) with quaternion-real matrix elements in the $2 \times 2$ Pauli basis $\left\{\sigma_{0}, \sigma_{X}, \sigma_{Y}, \sigma_{Z}\right\}$. The choice of the Pauli basis for the matrix elements induces a $2 N \times 2 N$ representation of $S$, which must satisfy (i) $S S^{\dagger}=S^{\dagger} S=I_{2 N}$ and (ii) $S=S^{R}$ where $S^{R}$ denotes the symplectic dual. Such constraints leave $2 N(N-1)$ real degrees of freedom for $S$.

In analogy to the prior case, we assume that the range of controls is restricted such that the Hamiltonian generating $S$ is always symplectic. Any symplectic dual transformation $S$ has the canonical representation $S=U^{R} U$ where $U \in U(2 N)$ [26]. Let $U_{1}, U_{2} \in U(2 N)$. The necessary and sufficient condition for $U_{1}^{T} U_{1}=U_{2}^{T} U_{2}$ is that $U_{1} U_{2}^{-1} \in S p(2 N)$ where $S p(2 N)$ denotes the real symplectic Lie group. The image of the mapping giving the canonical representation $U \rightarrow U^{R} U$ is homeomorphic 
to $U(2 N) / S p(2 N)$. Henceforth, we take $U(2 N) / S p(2 N)$ to be the space of symplectic unitary transformations over which we define as the landscape domain for the present case. In close analogy with the prior case, we may also define a target-invariant landscape function $\mathcal{J}[S]=\operatorname{Re} \operatorname{Tr}(S)$ defined over $U(2 N) / S p(2 N)$.

The first and second order analyses of the critical landscape topology can be replicated for the symplectic case by using, in analogy to Eq.(15), the canonical representation for unitary symplectic operators

$$
S=X^{R} \Omega X,
$$

with $X \in S p(2 N)$ and

$$
\Omega=\left(\begin{array}{lll}
\omega_{1} \sigma_{0} & & \\
& \ddots & \\
& & \omega_{N} \sigma_{0}
\end{array}\right),
$$

where $\omega_{1}, \ldots, \omega_{N}$ are the complex, unimodular eigenvalues of $S$.

Analogously to the prior case, there are $N+1$ critical submanifolds with the structure of symplectic Grassmannians. Collectively, the critical set is the union

$$
\bigcup_{n=0}^{N} G_{\text {symplectic }}(2 n, 2 N)
$$

where

$$
G_{\text {symplectic }}(2 n, 2 N)=S p(2 N) / S p(2 N-2 n) \times S p(2 n)
$$

embedded in $U(2 N) / S p(2 N)$, with dimension

$$
\operatorname{dim} G_{\text {symplectic }}(2 n, 2 N)=N(2 N-1)-(N-n)[2(N-n)-1]-n[2 n-1] .
$$

The HQF can be obtained similarly. Let us evaluate $\mathcal{J}$ at some critical point $\tilde{S}$ in the representation $\tilde{S}=X^{R} \tilde{\Omega} X$. In analogy to the prior case, we differentiate $\tilde{S}$ twice over the arc given by $\tilde{S} \rightarrow S^{\prime}=$ $\left.\frac{d \tilde{S}}{d t}\right|_{t=0}=\sqrt{\tilde{S}} i A \sqrt{\tilde{S}} \rightarrow S^{\prime \prime}=-\sqrt{\tilde{S}} A^{2} \sqrt{\tilde{S}}$ defined by some $A=A^{R}$ (where $A_{i j}=\alpha_{i j} \sigma_{0}+\beta_{i j} \sigma_{x}+$ 
$\gamma_{i j} \sigma_{y}+\delta_{i j} \sigma_{z}$ with real $\left.\alpha_{i j}, \beta_{i j}, \gamma_{i j}, \delta_{i j}\right)$ and evaluate the landscape function with the arc-parameterized argument to obtain, up to multiplicative constants

$$
\mathcal{H}_{\mathcal{J}}(S)=-\sum_{i<j}\left(\omega_{i}+\omega_{j}\right)\left(\alpha_{i j}^{2}+\beta_{i j}^{2}+\delta_{i j}^{2}+\gamma_{i j}^{2}\right)-\sum_{i} \omega_{i} \lambda_{i}^{2}
$$

The number of positive, negative and zero-valued elements of the HQF are, respectively,

$$
\begin{aligned}
& D_{+}=2(N-n)^{2}+2(N-n) \\
& D_{-}=2 n^{2}+2 n \\
& D_{0}=4 N(N-n) .
\end{aligned}
$$

\section{Conclusions}

The analysis reveals that the critical submanifolds for the symmetry-restricted landscapes are of two types: isolated points corresponding to the global maxima and minima, and Grassmannian submanifolds corresponding to the sub-optimal extrema values. Although the sub-optimal Grassmannian solutions are more numerous and more voluminous than the global solutions, the Hessian analysis reveals that all such local solutions have saddlepoint structure and thus do not act as local traps. Furthermore, the invariance of the qualitative landscape structure with respect to the target transformation demonstrated in this analysis suggests that no quantum unitary transformation should be any more difficult to optimally construct than another. In addition to prior works [9, 10, the present analysis is a strong endorsement of optimal search as a practically viable means of generating unitary transformations for complex systems, and demonstrates that the most important qualitative aspects of the landscape are not affected when certain symmetry restrictions are placed on the underlying dynamics. 


\section{REFERENCES}

[1] P. Brumer, M. Shapiro "Principles of Quantum Control of Molecular Processes" John Wiley \& Sons, (2003).

[2] M. A. Nielsen and I. L. Chuang, "Quantum Computation and Quantum Information", Cambridge University Press, (2000).

[3] S. Shi, H. Rabitz, Chem. Phys. 139, 185 (1989).

[4] G. Gordon, G. Kurizki, D.A. Lidar, Phys. Rev. Lett. 101, 010403 (2008). C. Meier, M. Heitz, J. Chem. Phys. 123, 044504 (2005). I. Serban, J. Werschnik, E. Gross, Phys. Rev. A 71, 053810 (2005). M. Sukharev, T. Seideman, Phys. Rev. Lett. 93, 093004 (2004). C. M. Tesch, R. de Vivie-Riedle, J. Chem. Phys. 121, 12158 (2004). E. Luc-Koenig, R. Kosloff, F. Masnou-Seeuws and M. Vatasescu, Phys. Rev. A 70, 033414 (2004). D. Babikov, J. Chem. Phys. 121, 7577 (2004). B. Lan, I. Vrabel, W. Jakubetz, J. Chem. Phys. 121, 10401 (2004). J. Palao, R. Kosloff, Phys. Rev. A 68, 062308 (2003). D. Geppert, A. Hoffman, R. de Vivie-Riedle, J. Chem. Phys. 119, 5901 (2003). T. Hornung, S. Gordienko, R. de Vivie-Riedle, B. Verhaar, Phys. Rev. A 66, 043607 (2002). J. Palao, R. Kosloff, Phys. Rev. Lett. 89, 188301 (2002). T. Mancal, U. Kleinekathofer, V. May, J. Chem. Phys. 117, 636 (2002). T. Hornung, M. Motzkus, R. de Vivie-Riedle, J. Chem. Phys. 115, 3105 (2001). Y. Ohtsuki, H. Kono, Y. Fujimura, J. Chem. Phys. 109, 9318 (1998).

[5] R. S. Judson, H. Rabitz Phys. Rev. Lett. 68, 1500 (1992).

[6] C. J. Bardeen et al., Chem. Phys. Lett. 280, 151 (1997). M. Bergt, T. Brixner, M. Strehle, G. Gerber, J. Phys. Chem. A. 103, 10381 1999. D. Meshulach, Y. Silberberg, Nature 396, 239 (1998). A. Assion et al., Science 282, 919 (1998). T. Hornung, R. Meier, D. Zeidler, K.-L. Kompa, D. Proch, M. Motzkus, Appl. Phys. B: Lasers Opt. 71, 277 (2000). T. Weinacht, J. White, P. H. Bucksbaum, J. Phys. Chem. A 103, 10166 (1999). R. Bartels et al. Nature 406,164 (2000). C. Daniel et al., Chem. Phys. 267, 247 (2001). T. Hornung et al., Chem. Phys. 267, 261 (2001). J. Kunde et al., J. Opt. Soc. Am. B 18, 872 (2001). T. Brixner, H. Damrauer, P. Niklaus, G. Gerber, Nature 414, 57 (2001). R. J. Levis, G. M. Menkir, H. Rabitz, Science 292, 709 (2001). M. Bergt et al., J. Organ. Chem. 661, 199 (2002). T. Brixner et al., J. Mod. Opt. 50, 539 (2003), E. Hertz et al., J. Chem. Phys. 118, 595 (2003).

[7] J. Palao, R. Kosloff, Phys. Rev. Lett. 89, 188301 (2002).

[8] C. Tesch, R. de Vivie-Riedle, Phys. Rev. Lett. 89, 157901 (2002). J. Palao, R. Kosloff, Phys. Rev. A 68, 062308 (2003). D. Babikov J. Chem. Phys. 121, 7577 (2004). S. Suzuki, K. Mishima, K. Yamashita Chem. Phys. Lett. 410, 358 (2005). Y.Ohtsuki Chem. Phys. Lett. 404, 126 (2005). U. Troppmann, R. de Vivie-Riedle J. Chem. Phys. 122, 154105 (2005).

[9] H. Rabitz, M. Hsieh, C. Rosenthal, Phys. Rev. A 72, 052337 (2005).

[10] M. Hsieh, H. Rabitz, Phys. Rev. A, 77, 042306 (2008). 
[11] J. von Neumann, Tomsk Univ. Rev., 1, 286 (1937).

[12] S. S. Cairns (ed.), "Differential and Combinatorial Topology" Princeton University Press (1965).

[13] L. Geissinger, Lin. Multilin. Alg., 5, 321 (1978).

[14] M. Shayman, Unpublished manuscript, (1982).

[15] R. Brockett, Lin. Alg. Appl., 122/123/124, 701 (1989).

[16] K. Moore. M. Hsieh, H. Rabitz, J. Chem. Phys., 128, 154117 (2008).

[17] F. Dyson, J. Math. Phys. 3, 140 (1962).

[18] R. Ernst, G. Bodenhausen, A. Wokaun, "Principles of Nuclear Magnetic Resonance in One and Two Dimensions" Oxford Science Publications (1987).

[19] N. E. Bonesteel, D. Stepanenko, D. P. DiVincenzo, Phys. Rev. Lett., 87, 207901 (2001).

[20] D. Stepanenko, N. E. Bonesteel, D. P. DiVincenzo, G. Burkard, D. Loss Phys. Rev. B, 68, 115306 (2003).

[21] E. Farhi and S. Gutmann Phys. Rev. A, 58, 915 (1998).

[22] A. Childs, E. Farhi, S. Gutmann Quant. Inf. Proc., 1, 35 (2002).

[23] G. Giedke and J. Ignacio Cirac Phys. Rev. A, 66, 032316 (2002).

[24] R. Simon, E. C. G. Sudarshan, N. Mukunda Phys. Rev. A, 37, 3028 (1988).

[25] C. E. Porter (Ed.), "Statistical Theories of Spectra: Fluctuations" Academic Press, (1965).

[26] M. L. Mehta, "Random Matrices" Academic Press, (1991).

[27] G. Banaszak, W. Gajda, P. Krason (Eds.), "Algebraic K-Theory" American Mathematical Society, (1996).

[28] L. Hua, "Harmonic Analysis of Functions of Several Complex Variables in the Classical Domains" American Mathematical Society, (1963).

[29] U. Helmke and J. Moore, "Optimization and Dynamical Systems" Springer Verlag, (1994).

[30] R. Horn and C. Johnson, "Matrix Analysis" Cambridge University Press, (1985).

[31] M. Demazure, "Bifurcations and Catastrophes: Geometry of Solutions to Nonlinear Problems" Springer Verlag (2000). 